\title{
An evaluation of the risk factors of breast cancer in women in Ilam Province: a case-control study based on hospital
}

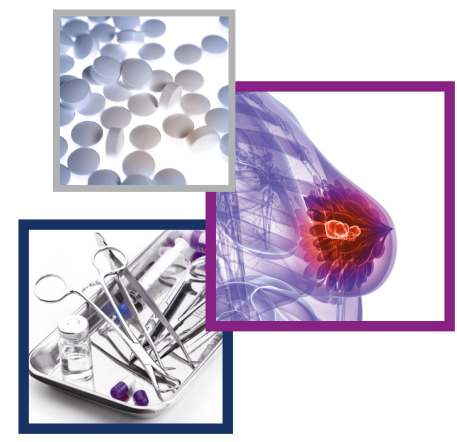

\author{
Shoboo Rahmati ${ }^{1}$ (D), Milad Azami ${ }^{2}$ (D), Zahra Jaafari ${ }^{3}$, Nasrin Pouratar ${ }^{1}$ \& Khairollah \\ Asodollahi*,4 (iD) \\ ${ }^{1}$ Department of Epidemiology, Student Research Committee, Ilam University of Medical Sciences, Ilam, Iran \\ ${ }^{2}$ Department of Medicine, Ilam University of Medical Sciences, Ilam, Iran \\ ${ }^{3}$ Student Research Committee, Kerman University of Medical Sciences, Kerman, Iran \\ ${ }^{4}$ Department of Epidemiology, Faculty of Medicine, Ilam University of Medical Sciences, Ilam, Iran \\ *Author for correspondence: masoud_1241@yahoo.co.uk
}

\begin{abstract}
Aims: The present study aimed to identify the most associated risk factors of breast cancer in llam province in 2015-2016. Materials \& methods: In this case-control study, the case group consisted of 50 women with breast cancer with a definitive diagnosis from date 23 September 2015 to 22 September 2016. The two groups were group matched in terms of age. Data were collected through interviews and medical records. Results: According to the results, there was a significant relationship between the disease status and variables of family history, history of irregular menstrual cycles, history of breastfeeding, menopausal status, history of oral contraceptive pill consumption, BMI, menarche and number of pregnancies ( $p<$ 0.05). Conclusion: Regarding decisive risk factors of breast cancer, more studies with larger sample sizes that take into account more risk factors are necessary.
\end{abstract}

First draft submitted: 13 May 2020; Accepted for publication: 23 July 2020; Published online:

18 September 2020

Keywords: breast cancer $\bullet$ Ilam $\bullet$ Iran $\bullet$ risk factors

\section{Background}

Breast cancer is one the most common types of cancer and the main cause of death from cancer in women worldwide. In 2012, around 1.7 million cases and 521,900 cases of death (i.e., 25\% of all cancer cases and 15\% of all mortality from cancer) occurred due to breast cancer [1]. Although breast cancer is the fifth highest cause of death from cancer worldwide. However, the most common cause of death from cancer among women in developing and developed countries has not been specified yet [2,3]. The incidence and mortality from breast cancer in 187 countries from 1980 to 2010 indicated that the incidence of breast cancer increased by $3.1 \%$ and the mortality from breast cancer increased by $1.8 \%$ every year [4]. The incidence and mortality from breast cancer in Iran follows the above mentioned trend. According to the latest report by national cancer registration system in 2008, breast cancer, with a prevalence of $24.8 \%$ of all cancers in women, is the most common type of cancer in women and constitutes $11.3 \%$ of all cancer incidences. Furthermore, it has an increasing trend in women; its crude incidence was 0.23 per 100,000 women in 2003-2004 and reached to 0.75 in 2008-2009. In addition, mean peak age of breast cancer in Iranian women is reported to be in their fourth and fifth decades of life, which is one decade lower than the global statistics [5] and doubles the importance of this issue for us. According to the latest report by the national cancer registration system in 2008, prevalence of breast cancer in Ilam province was around 12.04, which is considered a low prevalence along with provinces of Ardebil, Sistan and Baluchestan and Kohgiluyeh and Boyer-Ahmad [5]. Various risk factors are mentioned for breast cancer in different references including age (the incidence rate of breast cancer increases with age), genetic factors, family history of breast cancer, previous history of breast cancer, history of benign breast disease, early puberty before age 12 year, late menopause after age 55 year, previous history of chest radiation therapy and first delivery after age 30 year, obesity, alcohol consumption and consumption of high fat foods [6,7]. Since the above factors can be divided into two groups of modifiable and nonmodifiable factors,

Future Medicine 
it is important to identify the risk factors, particularly the modifiable factors. Identification of changeable factors provides the possibility to interfere and reduce the incidence of the disease, while identification of unchangeable factors provides the possibility to find the vulnerable groups and measures in primary and secondary prevention such as screening [8]. Therefore, the present study launched to identify the most associated risk factors of breast cancer via a case-control study.

\section{Method}

Materials \& methods

From 23 September 2015 to 22 September 2016, a case-control study was conducted among 100 women (50 women involving with breast cancer and 50 women without breast cancer) referred to obstetrics and gynecology clinics in Ilam province. The name, phone number and address of all subjects were obtained from the Health Center of Ilam province. The definitive diagnosis of breast cancer was done through biopsy and pathologic examinations in involved samples. For each subject with breast cancer, one patient hospitalized in Imam Khomeini hospital (Tehran, Iran), was selected through convenience sampling as a control. For the control group, patients without a breast disease or any malignancy were selected. The inclusion criteria for the case group included no history of chemotherapy, radiotherapy and hormone therapy, no history of benign breast mass, no history of hormone-related diseases (hyperthyroidism, polycystic ovary syndrome and irregular menstruation), grade 1 and 2 malignancy, no change in dietary patterns in the last 5 years, not being in the period of pregnancy or breastfeeding and no consumption of certain drugs such as methotrexate, cyclosporine, metformin, aspirin, epileptics and contraceptives [9]. Moreover, data collection and completion of the questionnaire was done by a trained midwife based on face to face interview. In case a subject passed away, one of the informed women of the family was interviewed. The questionnaire included questions about age, marital status, education, history of pregnancy, age of first menstruation, age of menopause, history of using oral contraceptives and history of breastfeeding. Written informed consent was obtained from all participants. Personal information and results of the data were set without mentioning the personal characteristics.

\section{Statistical analysis}

Data analysis was conducted by chi-square (Fisher's exact test), t-test and regression logistic on SPSS $_{21}$ software was used and significance level considered as 0.05 .

\section{Results}

In this case-control study, the case group consisted of 50 women with breast cancer with a definitive diagnosis based on pathology reports and eligible for the research and the control group included 50 women (hospital controls) referred to Imam Khomeini hospital without a neoplastic disease. Mean and standard deviation of age were $45.7 \pm 8.7$ and $45.2 \pm 11.3$ years in case and control groups, respectively. And illustrate the distribution of studied variables in both groups of case and control. Moreover, since age factor was a matched.

Data analysis showed that a significant relationship between breast cancer and family history, history of irregular menstrual cycles, history of breastfeeding, menopausal status, history of oral contraceptive consumption, BMI, menarche and number of pregnancies was revealed $(\mathrm{p}<0.05)$. However, no significant relationship was found between the disease status and variables of marital status, age of marriage, age of first pregnancy, history of abortion, income, place of residence, smoking and career $(\mathrm{p}>0.05$; Tables $1 \& 2)$.

Finally, the variables that showed a significant relationship with breast cancer and were appropriate for the model included: exercise, family history, irregular menstrual history, history of benign breast disease, history of breastfeeding, menopausal status, history of oral contraceptive pill (OCP) and BMI, Menarche and the number of pregnancies entered the multivariate logistic regression model. The results showed that controlling the effect of other factors like exercise, BMI, menarche, irregular menstrual history, history of benign breast disease, OCP Consumption history and breastfeeding history are important variables in predicting breast cancer in the subjects (Table 3).

\section{Discussion}

Breast cancer is the most common type of cancer among women and considering its risk factors and informing women about this disease is highly essential [10]. The analysis results demonstrated that women with family history of breast cancer had a greater chance of breast cancer. In a study entitled 'Risk factors of breast cancer in women under 40 years old' in Cancer Institute of Imam Khomeini hospital in Tehran, Tehranian et al. reported an odds 
Table 1. The frequency distribution of qualitative variables in both case and control groups of breast cancer.

Variable

\begin{tabular}{ll} 
Education level & Ynder diploma \\
\hline
\end{tabular}

\section{Smoking}

Job

Job

Location status

Marital status

Income status

History of irregular menstruation

History of breastfeeding

Menopause status

History of OCP use

History of abortion

Family history

Maternal age at the time of first

pregnancy

OCP: Oral contraceptive pill.
Disease

Yes

$37(52.1 \%)$

$13(44.8 \%)$

$3(6 \%)$

$47(94 \%)$

$0(0 \%)$

Current smoker

Housekeeper

Employee

Urban

Rural

Single

Married

Under 1 million toman Upper 1 million toman

Yes

No

Yes

No

Yes

No

No $\quad 12(26.7 \%)$

Yes $10(47.6 \%)$

No $\quad 35(54.7 \%)$

Yes $31(62 \%)$

No $19(38 \%)$

$>30$ years $\quad 15(60 \%)$

$<30$ years $\quad 30(50 \%)$

No

$34(47.9 \%)$

$16(55.2 \%)$

$1(2 \%)$

$49(98 \%)$

$0(0 \%)$

$39(45.9 \%)$

$11(73.3 \%)$

$40(80 \%)$

$10(20 \%)$

$10(20 \%)$

$40(80 \%)$

$30(51.7 \%)$

$9(46.3 \%)$

$16(30.8 \%)$

$34(70.8 \%)$

$33(66 \%)$

$7(20 \%)$

$16(32 \%)$

$34(68 \%)$

$11(27.5 \%)$

$29(72.5 \%)$

$11(52.4 \%)$

$29(45.3 \%)$

$19(38 \%)$

$31(62 \%)$

$10(40 \%)$

$30(50 \%)$ p-value

0.6

0.61

0.09

0.48

0.26

0.68

0.0001

0.0001

0.0001

0.0001

0.60

0.027

0.47

Table 2. Comparing the quantitative variables between case and control groups of breast cancer.

\begin{tabular}{|c|c|c|c|}
\hline & & Mean \pm SD & p-value \\
\hline \multirow[t]{2}{*}{ BMI } & Case & $27.6 \pm 3.6$ & 0.01 \\
\hline & Control & $25.46 \pm 4.4$ & \\
\hline \multirow[t]{2}{*}{ Menarche } & Case & $13.26 \pm 1.6$ & 0.0001 \\
\hline & Control & $14.66 \pm 1.23$ & \\
\hline \multirow[t]{2}{*}{ Number of pregnancy } & Case & $2.7 \pm 1.9$ & 0.02 \\
\hline & Control & $3.9 \pm 2.8$ & \\
\hline \multirow[t]{2}{*}{ Marriage age } & Case & $19.7 \pm 4.6$ & 0.2 \\
\hline & Control & $18.6 \pm 5$ & \\
\hline
\end{tabular}

Table 3. Multivariate logistic regression statistical analysis.

Variable OR

\begin{tabular}{|ll} 
Exercise & No \\
& Yes \\
\hline
\end{tabular}

BMI 1.2

Menarche

History of irregular menstruation

No 1

History of benign breast disease

Yes

0.002

1.2

4.6

4.6

No 1

History of OCP use

Yes 10

No 1

Yes 11.9

History of breastfeeding

No 1

$\begin{array}{ll}\text { Yes } & 0.04\end{array}$

$95 \% \mathrm{Cl}$

$(0.00-0.32)$

p-value

(1-2.3) $\quad 0.012$

(1.3-15.6)

0.013

$(1.3-8.2)$

0.026

$(3.1-69)$

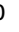

0.04

\begin{tabular}{ll}
$(3.1-69)$ & 0.031 \\
\hline$(1.5-24.1)$ & 0.019 \\
\hline$(0.002-0.8)$ & 0.042
\end{tabular}

OCP: Oral contraceptive pill; OR: Odds ratio. 
ratio (OR) of 7.07 in women with positive family history compared with those without family history [11]. Tavani et al. reported an OR of 1.85 in women with positive family history compared with those without family history [12]. Ebrahimi et al. in a study entitled 'Risk factors for breast cancer in Iran' demonstrated that risk factors in women with positive family history was more than others [13]. Jafarinia et al. in a study entitled 'Risk factors of breast cancer in Dezful' demonstrated that family history was one of the major risk factors of breast cancer [14]. The results of most of the studies in this field emphasized on the effect of family history as a risk factor for breast cancer, which are in line with the results of the present study.

Our study demonstrated that the risk of breast cancer was higher in women with lower age of menarche and this inverse relationship was significant according to univariate analysis. Tehranian et al. also demonstrated that the risk of breast cancer was higher in women with lower age of menarche [11]. Tavani $e t$ al. also demonstrated that age of menarche lower than 12 years was a risk factor of breast cancer [12]. However, Ebrahimi et al. reported that there was not a significant relationship between age of menarche and the risk of breast cancer [13]. Yankaskas et al. in a study entitled 'Epidemiology of breast cancer in young women', also reported an insignificant relationship between age of menarche and the risk of breast cancer [15]. Jafarinia et al. also demonstrated that there was not a significant relationship between age of the first menstruation (menarche) and risk of breast cancer [14]. The studies of Tehranian and Ebrahimi $e t$ al. were both conducted in Imam Khomeini Hospital in Tehran, and the first study confirmed a significant relationship between the age of menarche and risk of breast cancer but the second study reported a lack of significant relationship. In addition, our present study and some other studies confirmed the significance of the relationship, while some other studies did not confirm this significance and this inconsistency may be due to use of different methodologies applied in these studies. To prove the true relationship between the age of menarche and the risk of breast cancer, more accurate and prospective investigations with bigger sample sizes are required in the future.

The present study demonstrated that the mean number of pregnancies in case group was less than the control group and this relationship was significant according to univariate analysis, indicating that risk of breast cancer was lower in women with more pregnancies. Layde et al. in a study demonstrated that OR in women with more than seven pregnancies was 0.59 compared with nulliparous women (16). However, Tavani et al. demonstrated that $\mathrm{OR}$ in multiparous women under 40 years old was lower than nulliparous women [12]. Based on a univariate analysis, Tehranian et al. demonstrated that OR in women with more than four deliveries was 13.33 compared with nulliparous women [11]. These differences may be due to selection of samples with high mean age and using different methodologies. Proving the relationship between number of births and breast cancer requires more investigations.

The present study demonstrated that there was no significant relationship between age at the first birth and risk of breast cancer, which was in accordance with the study by Ebrahimi et al. Yankaskas et al. demonstrated that there was a significant relationship between age at the first birth and risk of breast cancer [15]. Tavani $e t$ al. also demonstrated that risk of breast cancer was higher in primiparous women older than 30 years [12]. This difference may be due to small sample size of the present study.

The present study also demonstrated that breastfeeding was a protective factor against breast cancer based on both univariate analyses. Jafarinia $e t$ al. also demonstrated that breastfeeding was a protective factor against breast cancer [14]. Toleutay et al. demonstrated that history of breastfeeding was a protective factor against breast cancer [17]. Layde $e t$ al. demonstrated that risk of breast cancer in women with history of breastfeeding longer than 25 months was lower than those with no history of breastfeeding [16]. Based on a study by Zheng et al. in China, risk of breast cancer in women with history of breastfeeding longer than 24 months was lower than those with history of 1-6 months breastfeeding [18]. In another study by Yuan et al. in China, risk of breast cancer in women with history of breastfeeding longer than 109 months was lower than those with no history of breastfeeding [19]. Study of Tehranian et al. demonstrated that the relative risk in women with history of breastfeeding was lower than those with no history of breastfeeding [11]. Similar to our study, several studies emphasized on the importance of breastfeeding as a protective factor for breast cancer.

The present study demonstrated that there was a significant relationship between taking contraceptive pills and risk of breast cancer and the prevalence rate of taking contraceptive pills among cases compared with controls was almost triple ( 73.3 vs $27.5 \%$, respectively). According to univariate analysis, the OR of taking contraceptive pills and the risk of breast cancer were 7.2 and 11.9, respectively, compared with control group. Tehranian $e t$ al. also demonstrated that there was a significant relationship in taking contraceptive pills and risk of breast cancer between the two groups. They reported a prevalence rate of taking contraceptive pills as 38.8 and $18.5 \%$, among case and control groups, respectively. Also based on their study, the OR in women who took contraceptive pills 
was 2.83 compared with those who did not [11]. Sidoni et al. also demonstrated that history of taking contraceptive pills increased the risk of breast cancer [20]. However, Ebrahimi et al. demonstrated that there was no significant relationship between taking contraceptive pills and risk of breast cancer [13]. Marchbanks et al. also demonstrated that there was no significant relationship between taking contraceptive pills and risk of breast cancer [21]. Most studies emphasized on the positive relationship between taking contraceptive pills and risk of breast cancer and lack of such reports in some studies might be due to using different methodologies.

According to our results, there was no significant relationship between history of abortion and breast cancer. Tehranian et al. demonstrated that relationship between history of abortion and breast cancer was not statistically significant [11]. According to a study by Mahue-Giangreco et al. the risk of breast cancer did not increase in young women with a history of abortion [22]. However, a study evaluating the relationship between history of abortion and breast cancer demonstrated that the risk of breast cancer in women with a history of induced abortion before 12th week of pregnancy was 2.1-26.2-times more than that among women without a history of induced abortion [23]. In addition, the risk of breast cancer in women with a history of spontaneous abortion after 12th week of pregnancy was 2.5-16.3-times more than that among women without a history of spontaneous abortion. The risk of breast cancer in women with a history of spontaneous abortion, whose the first abortion occurred after 12th week of pregnancy was 4.7-55.7-times more than that among women without a history of spontaneous abortion. Proving the relationship between history of abortion and breast cancer requires more investigations.

The present study demonstrated that risk of breast cancer was higher in women with higher BMI, which was in accordance with the study of Holakouie et al. [24].

One of the limitations of this study was recall bias and women in case group were more likely to recall past events than women in control group. Another limitation was small sample size.

\section{Conclusion}

The overall results of the current study demonstrated that there was a significant relationship between the disease status and variables of family history, history of irregular menstrual cycles, history of breastfeeding, menopausal status, history of OCP consumption, BMI, menarche and number of pregnancies.

\section{Authors contributions}

SHR, MA, ZJ, NP and KHA acquired in idea and data. SHR analyzed and interpreted the data. SHR and MA drafted the manuscript; SHR critically revised the manuscript for important intellectual content. SHR supervised the study. All authors have read and approved the manuscript.

\section{Acknowledgments}

We sincerely thank Ilam University of Medical Sciences for helping us with this research.

\section{Financial and competing interests disclosure}

The authors have no relevant affiliations or financialinvolvement with any organization or entity with a financial interest in or financial conflict with the subject matter or materials discussed in the manuscript. This includes employment, consultancies, honoraria, stock ownership or options, expert testimony, grants or patents received or pending, or royalties.

No writing assistance was utilized in the production of this manuscript.

\section{Ethical conduct of research}

The study was approved by llam University of Medical Sciences Ethical Review Committee; IR.MEDILAM.REC.1394.205.

\section{Open access}

This work is licensed under the Attribution-NonCommercial-NoDerivatives 4.0 Unported License. To view a copy of this license, visit http://creativecommons.org/licenses/by-nc-nd/4.0/

\section{References}

1. Ban KA, Godellas CV. Epidemiology of breast cancer. Surg. Oncol. Clin. N. Am. 23(3), 409-422 (2014).

2. DeSantis CE, Bray F, Ferlay J, Lortet-Tieulent J, Anderson BO, Jemal A. International variation in female breast cancer incidence and mortality rates. Cancer Epidemiol. Biomarkers Prev. 24(10), 1495-1506 (2015).

3. Jemal A, Center MM, DeSantis C, Ward EM. Global patterns of cancer incidence and mortality rates and trends. Cancer Epidemiol. Biomarkers Prev. 19(8), 1893-1907 (2010). 
4. Forouzanfar MH, Foreman KJ, Delossantos AM et al. Breast and cervical cancer in 187 countries between 1980 and 2010: a systematic analysis. Lancet 378(9801), 1461-1484 (2011).

5. Wu F, Guo Y, Chatterji S et al. Common risk factors for chronic non-communicable diseases among older adults in China, Ghana, Mexico, India, Russia and South Africa: the study on global AGEing and adult health (SAGE) wave 1. BMC Public Health 15(1), 88 (2015).

6. Wolff AC, Hammond MEH, Hicks DG et al. Recommendations for human epidermal growth factor receptor 2 testing in breast cancer: American Society of Clinical Oncology/College of American Pathologists clinical practice guideline update. Arch. Pathol. Lab. Med. 138(2), 241-256 (2014).

7. Azim HA, Michiels S, Bedard PL et al. Elucidating prognosis and biology of breast cancer arising in young women using gene expression profiling. Clin. Cancer Res. 18(5), 1341-1351 (2012).

8. Naghibi S-A, Shojaizadeh D, Montazeri A, Yazdani-Cherati J. Evaluating the performance rate of breast cancer screening methods and its relationship with breast cancer risk factors in Mazandaran province, Iran. J. Mazandaran Univ. Med. Sci. 23(110), 118-123 (2014).

9. Pirouzpanah S, Taleban F, Mehdipour P, Atri M, Hooshyareh-Rad A, Sabour S. The biomarker-based validity of a food frequency questionnaire to assess the intake status of folate, pyridoxine and cobalamin among Iranian primary breast cancer patients. Eur. J. Clin. Nutr. 68(3), 316 (2014).

10. Key TJ, Verkasalo PK, Banks E. Epidemiology of breast cancer. Lancet Oncol. 2(3), 133-40 (2001).

11. Tehranian N HpFTehranian N HpF. Hajizade A. Evaluating the Risk Factors breast cancer In women under 40 years. J. Tehran Univ. Med. Sci. 81, 37-44 (2009).

12. Tavani A, Gallus S, La Vecchia C et al. Risk factors for breast cancer in women under 40 years. Eur. J. Clin. Nutr. 35(9), 1361-1367 (1999).

13. Ebrahimi M, Vahdaninia M, Montazeri A. Risk factors for breast cancer in Iran: a case-control study. Breast Cancer Res. $4(5)$, R10 (2002).

14. Jafarinia B, Bahadorzai M, Delpisheh A, Sayehmiri K, Tavakoli M. Risk factors of breast cancer in Dezful City of Iran: a case-control study. Tehran Univ. Med. J. 74(2), 135-139 (2016).

15. Yankaskas BC. Epidemiology of breast cancer in young women. Breast Dis. 23(1), 3-8 (2006).

16. Layde PM, Webster LA, Baughman AL et al. The independent associations of parity, age at first full term pregnancy, and duration of breastfeeding with the risk of breast cancer. J. Clin. Epidemiol. 42(10), 963-973 (1989).

17. Toleutay U, Reznik V, Kalmatayeva Z, Smigelskas K. Risk factors of breast cancer in Kyzylorda oblast of Kazakhstan: a case-control study. Asian Pac. J. Cancer Prev. 14(10), 5961-5964 (2013).

18. Zheng T, Duan L, Liu Y et al. Lactation reduces breast cancer risk in Shandong Province, China. Am. J. Epidemiol. 152(12), 1129-1135 (2000).

19. Yuan JM, Mimi CY, Ross RK, Gao YT, Henderson BE. Risk factors for breast cancer in Chinese women in Shanghai. Cancer Res. 48(7), 1949-1953 (1988).

20. Sidoni A, Cavaliere A, Bellezza G, Scheibel M, Bucciarelli E. Breast cancer in young women: clinicopathological features and biological specificity. Breast 12(4), 247-250 (2003).

21. Marchbanks PA, McDonald JA, Wilson HG et al. Oral contraceptives and the risk of breast cancer. N. Engl. J. Med. 346(26), 2025-2032 (2002).

22. Mahue-Giangreco M, Ursin G, Sullivan-Halley J, Bernstein L. Induced abortion, miscarriage, and breast cancer risk of young women. Cancer Epidemiol. Biomarkers Prev. 12(3), 209-214 (2003).

23. Xiong NY, Zhou JH, Zhou XB, Zhang CY. Relationship between abortion and breast cancer on the basis of meta-analysis. BioMedical Information Engineering. 2009 International Conference on Future BioMedical Information Engineering 10.1109/FBIE.2009.5405887 (2009).

24. Holakouie Naeini K, Ardalan A, Mahmoudi M, Motevallian A, Yahyapour Y. Risk factors for breast cancer in Mazandaran Province, 2004. J. School Public Health Institute Public Health Res. 4(1), 27-36 (2006). 